\title{
Detección del primer caso de COVID-19 relacionado a la variante Ómicron en México
}

\author{
First COVID-19 case related to SARS-CoV-2 Omicron \\ variant detected in México
}

\begin{abstract}
Armando Rojo Enríquez, * Pilar Escamilla Llano,* Raymundo Rodríguez Sandoval, ${ }^{*}$ Leonardo Medina Arias, ${ }^{*}$ Miriam Ixchel García Franco, ${ }^{*}$ Leticia Ramírez De La O, ${ }^{*}$ Irma López Martínez, ${ }^{\ddagger}$ Claudia Wong Arámbula, ${ }^{\ddagger}$ Abril Rodríguez Maldonado, ${ }^{\ddagger}$ Vanessa Rivero Arredondo, ${ }^{\ddagger}$ Ariadna Medina Benítez, ${ }^{\ddagger}$ Joaquín Quiroz Mercado, ${ }^{\ddagger}$ Maribel González Villa, ${ }^{\ddagger}$ Natividad Cruz Ortiz, ${ }^{\ddagger}$ Tatiana Núñez García, ${ }^{\ddagger}$ Gisela Barrera Badillo, ${ }^{\ddagger}$ Fernando González Domínguez, ${ }^{\ddagger}$ Lucía Hernández Rivas, ${ }^{\ddagger}$ Ernesto Ramírez González ${ }^{\ddagger}$

Citar como: Rojo EA, Escamilla LP, Rodríguez SR, Medina AL, García FMI, Ramírez OL et al. Detección del primer caso de COVID-19 relacionado a la variante Ómicron en México. Acta Med GA. 2022; 20 (1): 62-66. https://dx.doi.org/10.35366/103558
\end{abstract}

\section{Resumen}

En noviembre de 2021 se informó sobre una nueva variante de preocupación (VoC) de SARS-CoV-2, la llamada variante Ómicron (B.1.1.529), que llamó la atención de los expertos por su elevado número de mutaciones en la proteína S. Presentamos a un paciente masculino de 51 años proveniente de Sudáfrica que comienza con síntomas respiratorios a las 48 horas de su llegada a la Ciudad de México. Durante su estancia en urgencias se obtuvo prueba de antígeno y RT-qPCR para SARS-CoV-2 positivas, con mínima alteración de parámetros de laboratorio y sin evidencia de afección pulmonar por tomografía. El paciente presentó evolución favorable y fue dado de alta seis días después. El día 29 de noviembre se recibió la notificación del Instituto de Diagnóstico y Referencia Epidemiológicos (InDRE) de que se trataba de la variante Ómicron del virus SARS-CoV-2.

Palabras clave: Ómicron, SARS-CoV-2, coronavirus, variantes, vacunación.

\section{Abstract}

On November 2021 a new SARS-CoV-2 variant of concern (VoC), named Omicron (B.1.1.529) was reported. This variant draw attention for its high number of mutations on the S protein. We present a 51 year old male, from South Africa who started with respiratory symptoms 48 hours after his arrival to Mexico City. During his stay at the ER a positive antigen and positive RT-qPCR were obtained. Minimal laboratory alterations were observed with no evidence of lung involvement on the CT scan. The outcome was favorable and was discharged six days later. On November 29, a notification from InDRE was received, reporting that the sequence obtained was compatible with SARS-CoV-2 Omicron variant.

Keywords: Omicron, SARS-CoV-2, coronavirus, variants, vaccination.
* Hospital Angeles Lomas. Ciudad de México, CDMX.

‡ Instituto de Diagnóstico y Referencia Epidemiológicos (InDRE). Ciudad de México, CDMX.

www.medigraphic.com/actamedica
Correspondencia:

Dr. Raymundo Rodríguez Sandoval

Correo electrónico: molecular.gass@gmail.com

Aceptado: 12-01-2022.

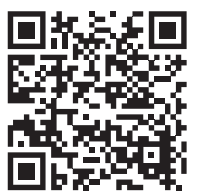




\section{INTRODUCCIÓN}

El 24 noviembre del 2021, a casi dos años del primer caso reportado de COVID-19 en Wuhan, China, hubo alrededor de 260 millones de casos confirmados y más de cinco millones de muertes en todo el mundo; ${ }^{1}$ la Organización Mundial de la Salud informó sobre una nueva variante de preocupación (VoC) de SARS-CoV-2, Ilamada Ómicron (B.1.1.529), la segunda en esta clasificación después de Delta. Esta variante presenta un número muy elevado de mutaciones, aproximadamente 32 en la proteína de la espícula (S), la región más antigénica y contra la cual se generan anticuerpos tanto por la vacuna como por infección. ${ }^{2}$ El primer caso identificado proviene de Botsuana, reportado el 11 de noviembre del 2021 y pocos días después otro caso más se originó en Hong Kong en un viajero proveniente de Sudáfrica. ${ }^{1}$ De acuerdo con las investigaciones, esta nueva variante presenta una "muy inusual constelación de mutaciones", por lo que ha causado preocupación en cuanto a si es capaz de evadir la inmunidad generada, ya sea por infección o por vacunación. ${ }^{3}$ A continuación, presentamos el primer caso confirmado de COVID-19 provocado por la variante Ómicron en México.

\section{PRESENTACIÓN DEL CASO}

Se trata de un paciente masculino de 51 años, sin antecedente de enfermedades crónico-degenerativas ni alergias. Presenta un esquema de vacunación completo contra SARS-CoV-2 por Pfizer-BioNTech con dos dosis. Originario y proveniente de Sudáfrica con llegada a la Ciudad de México el día 28 de noviembre del 2021. Dicho paciente tenía un antecedente de prueba negativa por reacción en cadena de la polimerasa (PCR) para SARS-CoV-2 realizada en su país de origen antes de viajar.

El cuadro clínico comenzó el día de su llegada a México con malestar general, mialgias, cefalea, congestión nasal y tos productiva, por lo que acudió al servicio de urgencias de nuestro hospital el 29 de noviembre del 2021. Durante su evaluación, el paciente se encontraba estable, sin alteraciones clínicas de relevancia. Signos vitales: FC 85 lpm, FR 18 rpm, temperatura $36.5{ }^{\circ} \mathrm{C}$ y saturación $\mathrm{O}_{2}$ 95\% al aire ambiente. Laboratorios (biometría hemática, química sanguínea de 27 elementos, interleucina 6, dímero D) sin alteraciones de relevancia, presentando únicamente proteína C reactiva $0.800 \mathrm{mg} / \mathrm{dL}(0.010-0.744 \mathrm{mg} / \mathrm{dL})$, ferritina $324.5 \mathrm{ng} / \mathrm{mL}(21.8-274.7 \mathrm{ng} / \mathrm{mL})$.

Anticuerpos IgG anti-nucleocápside SARS-CoV-2 negativos 0.09 índice ( $<1.4$ negativos). Anticuerpos IgM anti-nucleocápside SARS-CoV-2 negativos 0.33 índice $(<$

Figura 2: Curvas de amplificación para SARS-CoV-2 por RT-qPCR por Altona Diagnostics. La curva en color verde corresponde al control interno (Ct 35) y la curva azul corresponde al gen $\mathrm{E}$ (Ct 37). No se observa curva de amplificación del gen $\mathrm{S}$ al que correspondería una curva morada.

$\mathrm{RFU}=$ Relative Fluorescence Units.

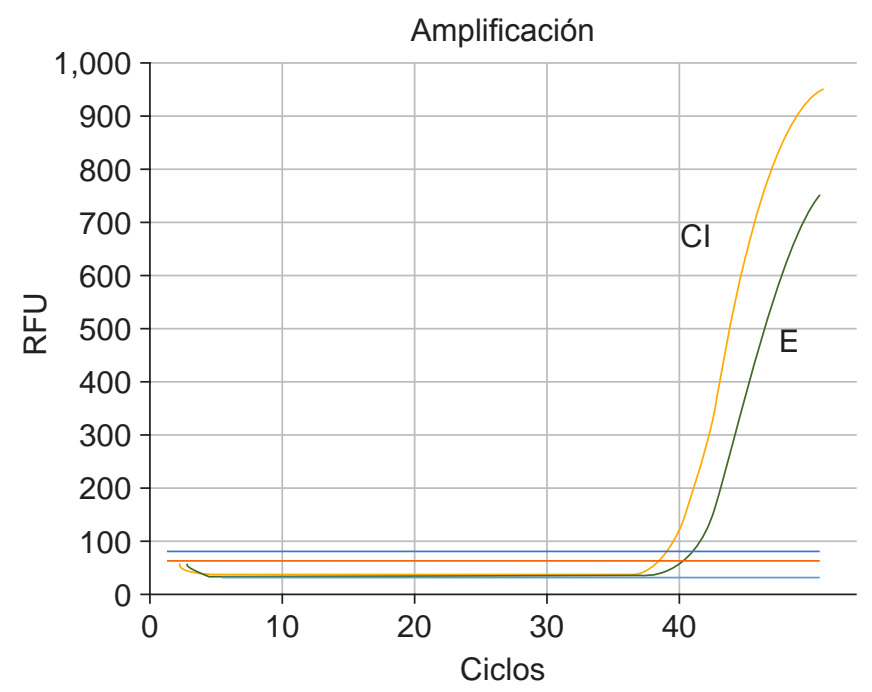

Figura 1:

Tomografía de tórax (corte axial y coronal) sin evidencia de afección pulmonar.
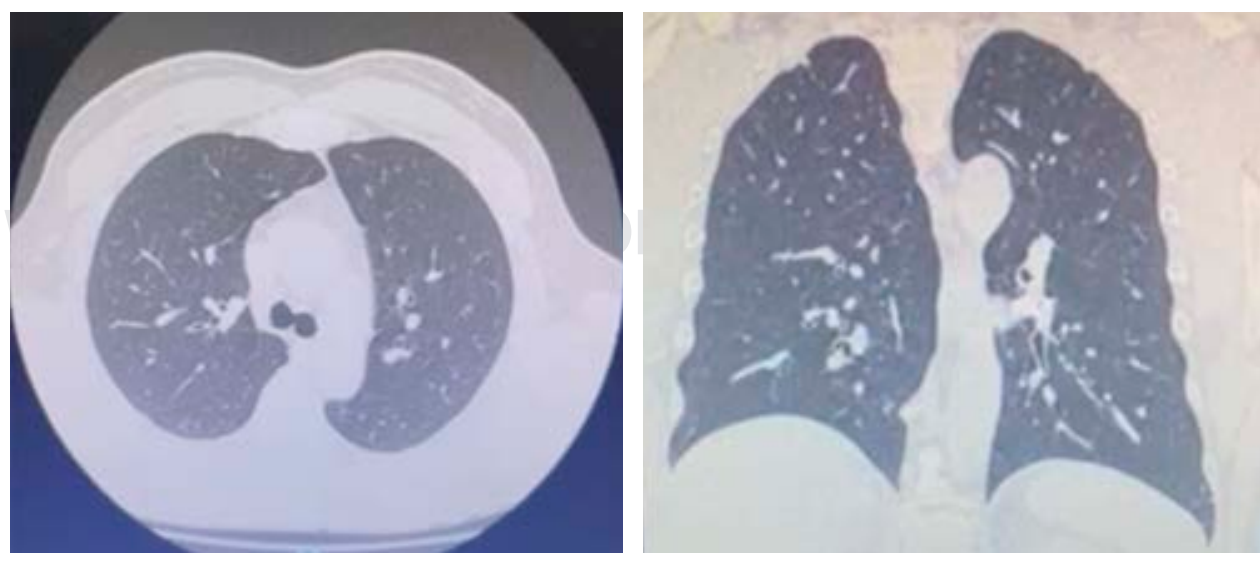
1 negativos). Anticuerpos IgG anti-proteína S SARS-CoV-2 positivos $20193.2 \mathrm{UA} / \mathrm{mL}$ (> $50 \mathrm{UA} / \mathrm{mL}$ positivos). Tomografía de tórax sin evidencia de afectación del parénquima pulmonar (Figura 1).

Se solicitó muestra de hisopado nasofaríngeo a la que se realizó prueba de detección de antígeno para SARSCoV-2 (Sofia, SARS antigen FIA, QUIDEL) con resultado positivo. Posteriormente, se realizó RT-qPCR confirmatoria utilizando las sondas RealStar ${ }^{\circledR}$ SARS-CoV-2 RT-qPCR Kit 1.0, Altona diagnostics observándose únicamente amplificación del gen E perteneciente a la familia de los betacoronavirus y ausencia del gen $\mathrm{S}$ específico para SARS-CoV-2 (Figura 2).

Dado que no se pudo corroborar el resultado positivo por la falta de amplificación de ambos genes, se procedió a realizar otra prueba RT-qPCR utilizando dos diferentes métodos. Uno fue el kit VIASURE SARS-CoV-2/Real Time $P C R$ Detection y el segundo el Xpert ${ }^{\circledR}$ Xpress SARS-CoV-2, GeneXpert en el cual se obtuvo un resultado positivo satisfactorio por la amplificación completa de los genes deseados; ORF1b y N para VIASURE y los genes E y N para GeneXpert (Figuras 3 y 4 ).

Ante la sospecha de la presencia de probables mutaciones en el gen $\mathrm{S}$ características de la variante Ómicron y teniendo en cuenta el antecedente epidemiológico del paciente, se solicitó secuenciación de la muestra al Instituto de Diagnóstico y Referencia Epidemiológicos (InDRE) para confirmación de la variante.

Figura 3: Curvas de amplificación de los genes ORF1b y $\mathrm{N}$ para SARS-CoV-2 mediante RT-qPCR kit VIASURE; la curva naranja corresponde al gen E (Ct 30) y la curva azul corresponde al gen ORF1b (Ct 32). El control interno pertenece a la curva verde.

$\mathrm{RFU}=$ Relative Fluorescence Units.

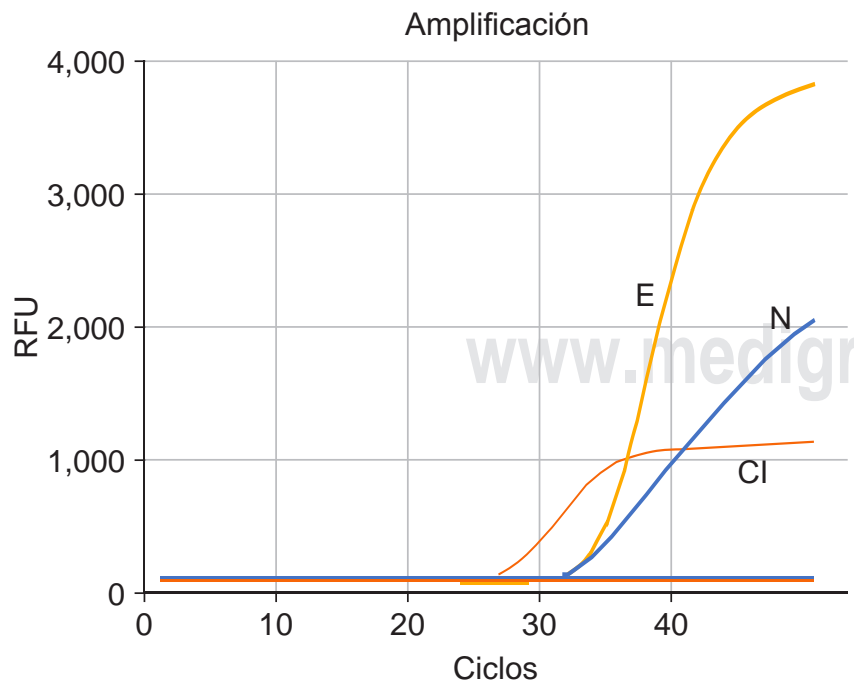

Figura 4: Curvas de amplificación de los genes $\mathrm{E}$ y N para SARS-CoV-2 mediante RT-qPCR GeneXpert; la curva azul corresponde al gen $\mathrm{E}$ (Ct 17) y la curva naranja corresponde al gen N (Ct 19).

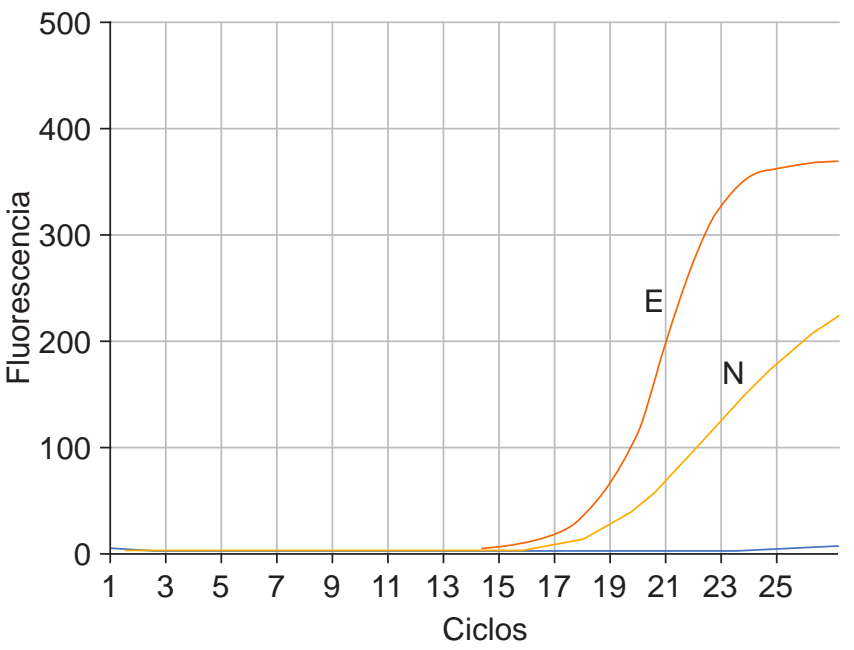

En cuanto al paciente, se solicitó ingreso hospitalario para aislamiento y contención de la transmisión por la alta sospecha de ser portador de la variante Ómicron. Durante su estancia hospitalaria recibió manejo con remdesivir por cinco días y antiinflamatorios no esteroideos (AINE), sin requerimiento de oxígeno suplementario $\left(\mathrm{SaO}_{2}\right.$ mayor a 95\%). Se mantuvo estable, afebril, refiriendo sólo fatiga y cefalea con buena respuesta a AINE. Normalización de estudios de laboratorio y radiografía de tórax sin cambios con respecto a la de ingreso. Al sexto día de estancia hospitalaria (día 9 de inicio de la sintomatología) se realizó nueva prueba de RT-qPCR en hisopado nasofaríngeo para SARSCoV-2 como seguimiento, donde se observó reducción importante de la carga viral (Ct 32/45). Por mejoría clínica y ante la ausencia de complicaciones se decide su egreso para continuar vigilancia y recuperación ambulatoria.

El día 29 de noviembre de 2021 se recibió confirmación oficial por parte del InDRE de que se trataba de la variante Ómicron y del primer caso identificado en el país (Figura 5).

\section{DISCUSIÓN}

Casi a diario se presentan nuevas variantes de SARSCoV-2 y de acuerdo con la Organización Mundial de la Salud, se clasifican en variantes de bajo monitoreo (VBM), variantes de interés $(\mathrm{Vol})$, variantes de preocupación $(\mathrm{VoC})$ y variantes de grandes consecuencias $(\mathrm{VoHC})$. La falta de una correcta política de salud, bajos índices de vacunación a nivel mundial, la existencia de una importante población de pacientes con diferentes tipos de inmunocompromiso y la nula vigilancia genómica del virus en países de 


\begin{tabular}{|c|c|}
\hline Virus name: & BCoV-19Mexico/CMX-InDRE FD122437_S9550/2021 \\
\hline Accession ID: & EPI ISL. 7156454 \\
\hline Type: & betacoronavirus \\
\hline Clade: & GR \\
\hline Pango L.incage: & B.1.1.529 (Pango v.3.1.16 2021-11-25), Omicton (B.1.1.529-like) (Scorpio) \\
\hline AN Substitutions: & 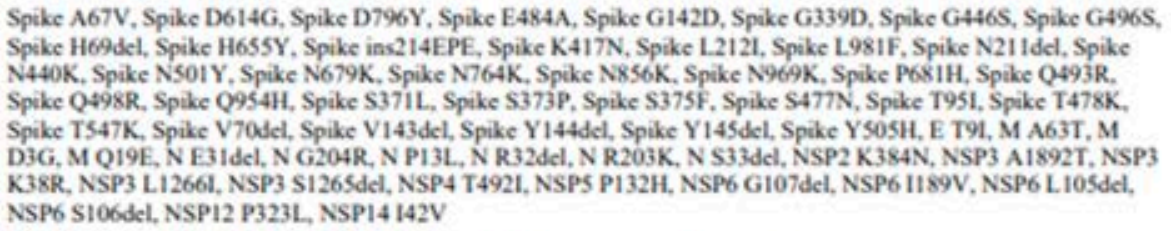 \\
\hline Variant: & VOC Omicron GR/484A (B.1.1.529) first detected in Botswana/Hong Kong South Africa \\
\hline Passage detailshistery: & Original \\
\hline \multicolumn{2}{|l|}{ Sample information } \\
\hline Collection date: & $2021-11-29$ \\
\hline Lecation: & North America / Mexico / Mexico City \\
\hline Host: & Human \\
\hline $\begin{array}{l}\text { Additional location } \\
\text { information: }\end{array}$ & Travel history: Africa \\
\hline Gender: & Male \\
\hline Paticat age: & 51 \\
\hline Paticat status: & Live \\
\hline Specimen source: & Nasopharyngeal swab \\
\hline \multicolumn{2}{|c|}{ Additional hest information: } \\
\hline \multicolumn{2}{|l|}{ Sampling strategy: } \\
\hline \multicolumn{2}{|l|}{ Outbreak: } \\
\hline Last vaccinated: & Pfizer, 2 doses \\
\hline \multicolumn{2}{|l|}{ Treatment: } \\
\hline Sequencing technology: & Illumina Nextseq \\
\hline Assembly method: & CL.C Genomics Workbench 21 \\
\hline Coverage: & $3414 x$ \\
\hline Comment: & $\begin{array}{l}\text { Insertion of } 6 \text { nucleotides when compared to the reference WIV04 sequence. Gap of } 36 \text { nucleotides when compared } \\
\text { to the reference WTV04 sequence. }\end{array}$ \\
\hline \multicolumn{2}{|l|}{ Institute information } \\
\hline Originating lab: & Hospital Ángeles Lomas \\
\hline Address: & Vialidad de la Barranca 240, Hacienda de las Palmas, C.P. 52763 Ciudad de México \\
\hline $\begin{array}{l}\text { Sample ID gives by the } \\
\text { originating laberatery: }\end{array}$ & 122437 \\
\hline Submitting lab: & Instituto de diagnóstico y Referencia Epidemiologicos (INDRE) \\
\hline Address: & $\begin{array}{l}\text { Francisco de P. Miranda } 177 \text { bis Colonia Lomas de Plateros Delegacion Alvaro Obregon, C. P. 01480, Mexico City, } \\
\text { Mexico. }\end{array}$ \\
\hline Sample ID given by the & 122437 \\
\hline
\end{tabular}

Figura 5: Reporte del InDRE confirmando el primer caso de COVID-19 relacionado a la variante Ómicron en México en el portal GISAID.

bajos recursos, representan las condiciones ideales para la generación de nuevas variantes y que éstas no sean detectadas a tiempo. La evidencia hasta este momento nos dice que, si bien la variante Ómicron puede ser mucho más contagiosa que las variantes previas, parece no producir cuadros severos de la enfermedad, como se ha presentado en las variantes anteriores (Tabla 1). ${ }^{4}$ Es recomendable llevar a cabo iniciativas de vigilancia genómica de una manera sistemática para poder estudiar las mutaciones y la evolución del virus. ${ }^{3}$ Es por esto que debe hacerse un esfuerzo a nivel global entre gobiernos, empresas farmacéuticas e instituciones de salud para poder controlar esta pandemia de manera efectiva. Se necesita más tiempo para poder tomar decisiones sobre los riesgos que representa esta nueva variante, aunque la inmunización masiva y las medidas de protección comunitaria seguirán siendo prioridad. Las autoridades gubernamentales deberán fortalecer las políticas y estrategias para conseguir la mayor cantidad de personas vacunadas. 
Tabla 1: Variantes de preocupación identificadas tanto en México como a nivel global y las repercusiones que las mutaciones tienen en la conducta del virus.

\begin{tabular}{|c|c|c|c|c|}
\hline Linaje Pango & Sinónimo & Origen & $\begin{array}{c}\text { No. de } \\
\text { mutaciones } \\
\text { en la espícula }\end{array}$ & Fenotipo \\
\hline Alpha & $\begin{array}{l}\text { B.1.1.7, } \\
\text { 20I/501Y.V1, } \\
\text { VOC } 202012 / 01\end{array}$ & $\begin{array}{l}\text { UK, Sept. } \\
2020\end{array}$ & 9 & $\begin{array}{l}\text { Mayor transmisibilidad: } 29 \% \text {. Aumento potencial del riesgo } \\
\text { de hospitalización (40-64\%) y riesgo de muerte (30-70\%). } \\
\text { Sin efectos adversos sobre la eficacia de la vacuna }\end{array}$ \\
\hline Beta & $\begin{array}{c}\text { B.1.351, } \\
\text { 20H/501Y.V2 }\end{array}$ & $\begin{array}{l}\text { Sudáfrica, } \\
\text { Ago. } 2020\end{array}$ & 9 & $\begin{array}{l}\text { Mayor transmisibilidad: } 25 \% \text {. Aumento potencial del } \\
\text { riesgo de muerte durante la hospitalización (20\%). } \\
\text { Impacta el escape inmunológico después de la infección } \\
\text { y después de la vacunación }\end{array}$ \\
\hline Gamma & $\begin{array}{l}\text { P.1, B.1.1.28.1, } \\
\text { 20J/501Y.V3 }\end{array}$ & $\begin{array}{l}\text { Brasil, Jul. } \\
2020\end{array}$ & 12 & $\begin{array}{l}\text { Mayor transmisibilidad: } 38 \% \text {. No se informó ningún } \\
\text { impacto sobre la gravedad de la enfermedad. Impacto } \\
\text { en el escape inmunológico después de la infección y } \\
\text { después de la vacunación }\end{array}$ \\
\hline Delta & $\begin{array}{c}\text { B.1.617.2, } \\
21 \mathrm{~J}\end{array}$ & $\begin{array}{l}\text { India, Dic. } \\
2020\end{array}$ & 10 & Mayor transmisibilidad: $97 \%$ \\
\hline Ómicron & $\begin{array}{l}\text { BA.1 (antes } \\
\text { B.1.1.529), } \\
21 \mathrm{~K}\end{array}$ & $\begin{array}{l}\text { Sudáfrica, } \\
\text { Dic } 2021\end{array}$ & 32 & Sin datos \\
\hline
\end{tabular}

\section{REFERENCIAS}

1. Karim SSA, Karim QA. Omicron SARS-CoV-2 variant: a new chapter in the COVID-19 pandemic [published online ahead of print, 2021 Dec 3]. Lancet. 2021;398(10317):2126-2128. doi: 10.1016/S01406736(21)02758-6.
2. Chen J, Wang R, Gilby NB, Wei GW. Omicron (B.1.1.529): Infectivity, vaccine breakthrough, and antibody resistance. J Chem Inf Model. 2022.

3. Kandeel M, Mohamed MEM, Abd El-Lateef HM, Venugopala KN, El-Beltagi HS. Omicron variant genome evolution and phylogenetics. J Med Virol. 2021. doi: 10.1002/jmv.27515.

4. ViralZone [Internet]. ViralZone; SARS-CoV-2, Variantes [citado 24 dic 2021]. Available in: https://viralzone.expasy.org/9556 